\title{
Fluctuations, correlations and the sign problem in QCD
}

\section{P. Lombardo*}

INFN Laboratori Nazionali di Frascati, I-00044, Frascati (RM), Italy

E-mail: lombardo@lnf.infn.it

\section{K. Splittorff}

The Niels Bohr Institute, Blegdamsvej 17, DK-2100, Copenhagen Ø, Denmark

E-mail: split@nbi.dk

\section{J.J.M. Verbaarschot}

Department of Physics and Astronomy, SUNY, Stony Brook, New York 11794, USA

E-mail: jv@chi.physics.sunysb.edu

\begin{abstract}
We study the distribution of the phase angle and the magnitude of the fermion determinant as well as its correlation with the chiral condensate and the baryon number for QCD at non-zero quark chemical potential. Results are derived to one-loop order in Chiral Perturbation Theory (ChPT), as well as by analytical and numerical calculations in QCD in one Euclidean dimension. We find a qualitative change of the distribution of the phase of the fermion determinant when the quark mass enters the spectrum of the Dirac operator: it changes from a periodicized Gaussian distribution to a periodicized Lorentzian distribution. We also explore the possibility that some observables remain weakly correlated with the phase of the fermion determinant even though the sign problem is severe. We discuss the practical implications of our findings on lattice simulations of QCD at non-zero baryochemical potential.
\end{abstract}

The XXVII International Symposium on Lattice Field Theory

July 26-31, 2009

Peking University, Beijing, China

\footnotetext{
* Speaker.
} 


\section{Introduction}

It is well known that standard lattice simulations based on importance sampling are not possible for QCD at non-zero chemical potential, since the measure in the functional integral is no longer positive definite. In the past years a few methods have been proposed to circumvent this problem, at least for small values of $\mu / T$ [1]. They all share a common philosophy: choose wisely a simulation ensemble - either at zero or non-zero imaginary chemical potential and fluctuations will allow the exploration of the target ensemble at non-zero baryon density. In a natural way, we are thus lead to consider the overlap between the simulation ensemble and the target ensemble, and to devise strategies to maximise it. This note, which is based on a more extended publication [2], reports our first results towards this goal.

Fluctuations increase at high temperature and in the vicinity of critical points, so in general ensembles generated close to $T_{c}$ allow a better reweighting [3], an easier calculation of the Taylor coefficients[4], or a safer extrapolation from imaginary chemical potential[5, 6, 7, 8]. Further, the shape of the distribution function plays a major role in the density of states method $[9,10]$ and some assumptions on its shape are needed.

The main goal of our investigation is to put these heuristic considerations on quantitative grounds by a combination of analytic studies and numerical simulations of chiral perturbation theory and QCD in one dimension.

We will base our discussion mostly on the $\theta$ distribution $\left\langle\delta\left(\theta-\theta^{\prime}\right)\right\rangle_{N_{f}} d \theta$, which will help assessing the overlap between simulation and target ensembles, and on the constrained distribution $\left\langle\mathscr{O} \delta\left(\theta-\theta^{\prime}\right)\right\rangle$ which shows how averages are built up in the spirit of the density of states method, since the integral over $\theta$ obviously gives the full expectation value $\langle\mathscr{O}\rangle$. The distribution of the observable with the phase allows us to address which range of the phase is essential for the full expectation value of $\mathscr{O}$.

\section{Results from Chiral Perturbation Theory}

Let us first recall that the $\theta$ distribution in the full theory

$$
\left\langle\delta\left(\theta-\theta^{\prime}\right)\right\rangle_{N_{f}} d \theta=\frac{\int d A\left|\operatorname{det}\left(D+\mu \gamma_{0}+m\right)\right|^{N_{f}} e^{i N_{f} \theta^{\prime}} \delta\left(\theta-\theta^{\prime}\right) e^{-S_{\mathrm{YM}}}}{\int d A\left|\operatorname{det}\left(D+\mu \gamma_{0}+m\right)\right|^{N_{f}} e^{i N_{f} \theta^{\prime}} e^{-S_{\mathrm{YM}}}} d \theta
$$

has a simple expression in terms of the phase quenched distribution

$$
\left\langle\delta\left(\theta-\theta^{\prime}\right)\right\rangle_{N_{f}}=e^{i \theta N_{f}} \frac{Z_{\left|N_{f}\right|}}{Z_{N_{f}}}\left\langle\delta\left(\theta-\theta^{\prime}\right)\right\rangle_{\left|N_{f}\right|} .
$$

In the following, we will mostly consider the two flavor case $\left(Z_{1+1^{*}} \equiv Z_{|2|}\right.$ and $\left.Z_{1+1} \equiv Z_{2}\right)$ :

$$
\left\langle\delta\left(\theta-\theta^{\prime}\right)\right\rangle_{1+1}=e^{2 i \theta} \frac{Z_{1+1^{*}}}{Z_{1+1}}\left\langle\delta\left(\theta-\theta^{\prime}\right)\right\rangle_{1+1^{*}} .
$$

The calculations follow the general method of [11]: the $\theta$ distribution is obtained from the moments of the phase factor

$$
\left\langle\delta\left(\theta-\theta^{\prime}\right)\right\rangle_{N_{f}}=\frac{1}{2 \pi} \sum_{p=-\infty}^{\infty} e^{-i p \theta}\left\langle e^{i p \theta^{\prime}}\right\rangle_{N_{f}}
$$



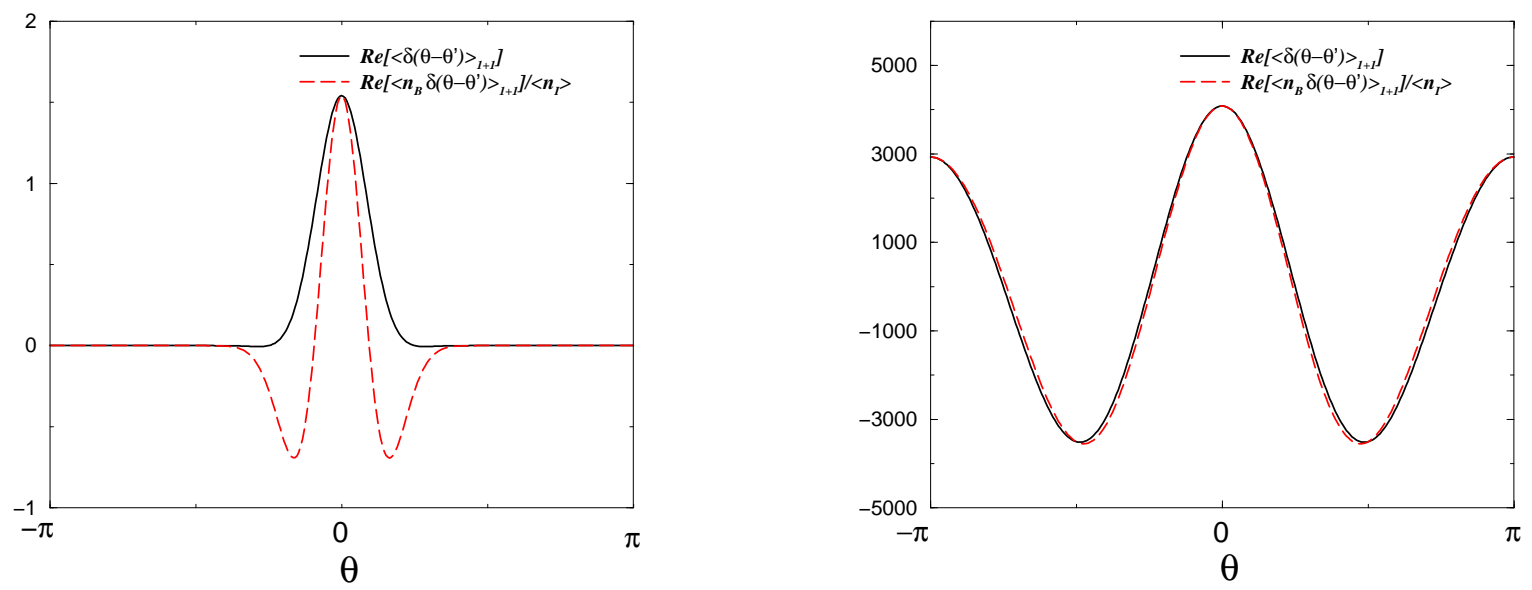

Figure 1: The real part of the distribution of the phase $\left\langle\delta\left(\theta-\theta^{\prime}\right)\right\rangle_{1+1}$ (solid curve) for a small $\Delta G_{0}=0.2$ (left) and a large $\Delta G_{0}=10$ value (right). Also shown is the real part of the distribution of the baryon number over $\theta$ (dashed curve). In either cases the $\theta$ distribution is normalised to 1 , while the integral of distribution of the baryon over $\theta$ is zero. This directly illustrates the severity of the sign problem at large $G_{0}$

First we consider $\mu<m_{\pi} / 2$ where the leading order difference between the phase quenched and full free energies is determined by the 1-loop corrections.

The $\theta$ distribution in one-loop ChPT for $\mu<m_{\pi} / 2$ is given by a periodicized Gaussian

$$
\left\langle\delta\left(\theta-\theta^{\prime}\right)\right\rangle_{1+1}=\frac{e^{2 i \theta}}{\sqrt{\pi \Delta G_{0}}} \sum_{n=-\infty}^{\infty} e^{-(\theta+2 \pi n)^{2} / \Delta G_{0}+\Delta G_{0}} .
$$

where $G_{0}$ is the free energy difference between neutral and charged pions. The distribution of the baryon number $n_{B}$ over $\theta$ is

$$
\left\langle n_{B} \delta\left(\theta-\theta^{\prime}\right)\right\rangle_{1+1}=\left(\lim _{\tilde{\mu} \rightarrow \mu} \frac{d}{d \tilde{\mu}} \Delta G_{0}(-\mu, \tilde{\mu})\right) \sum_{n=-\infty}^{\infty}\left(1+i \frac{\theta+2 \pi n}{\Delta G_{0}}\right) \frac{e^{2 i \theta}}{\sqrt{\pi \Delta G_{0}}} e^{-(\theta+2 \pi n)^{2} / \Delta G_{0}+\Delta G_{0}} .
$$

Note that the integral of the above distribution should be zero, since $\left\langle n_{B}\right\rangle=0$ in ChPT. In contrast the integral of $\theta$ distribution is unity. The similarity of the two distributions displayed in Fig. 1 clearly shows that the details of the distribution are important to get the correct results for $n_{B}$, especially when $G_{0}$ is large, i.e. when the sign problem is severe.

In order to make contact between these calculations and lattice studies, we have also computed the phase quenched as well as the partially quenched $\theta$ distribution where the ensemble is generated at zero chemical potential [12]. The relationship between the $\theta$ distributions derived in 1-loop ChPT is surprisingly simple: Whether we compute the width of the Gaussian for the $\theta$-distribution in the full ensemble generated at $\mu$, or the partially quenched ensemble generated at $\mu=0$, or in the quenched ensemble, or the phase quenched ensemble, we find exactly the same result. These results directly apply to the interpretation of lattice studies at small chemical potential $[12,13]$. 
We now proceed to the computation of the $\theta$-distribution for $\mu>m_{\pi} / 2$, this time derived from the even moments according to

$$
\left\langle\delta\left(2 \theta-2 \theta^{\prime}\right)\right\rangle_{N_{f}}=\frac{1}{\pi} \sum_{p=-\infty}^{\infty} e^{-2 i p \theta}\left\langle e^{2 i p \theta^{\prime}}\right\rangle_{N_{f}}
$$

The leading order contribution now enters already at mean field level. This leads to the result

$$
\left\langle\delta\left(2 \theta-2 \theta^{\prime}\right)\right\rangle_{1+1}=e^{2 i \theta} \frac{e^{V L_{B}}}{\pi} \frac{\sinh \left(V L_{B}\right)}{\cosh \left(V L_{B}\right)-\cos (2 \theta)},
$$

where $L_{B}$ is the difference of the free energy densities in the full and the phase quenched theory. The quenched result is obtained simply removing the general factor $e^{2 i \theta} e^{V L_{B}}$. In both cases the distribution is a periodicized Lorentzian. This is in sharp contrast with Gaussian obtained at low $\mu$, and implies that the hypothesis leading to the central limit theorem are not realised for $\mu>m_{\pi} / 2$.

Further, we discuss the the distribution of

$$
F=\left|\operatorname{det}\left(D+\mu \gamma_{0}+m\right)\right| / \operatorname{det}(D+m) \equiv \exp (f) .
$$

$F$ was studied in lattice QCD [12] using the Taylor expansion method. We have verified the assumption [12] that the $\theta$ distribution remains Gaussian even for a fixed value of $F$. Of course this applies only to $\mu<m_{\pi} / 2$.

Since determinants fluctuate by many orders of magnitude we feel that it is more appropriate to analyse the distribution of $\log F \equiv f$ instead. The two distributions are related by a simple transformation

$$
\left\langle\delta\left(f-f^{\prime}\right)\right\rangle=F\left\langle\delta\left(F-F^{\prime}\right)\right\rangle .
$$

The fluctuations of $f$ are induced by the gauge field fluctuations.

We find that for $\mu<m_{\pi} / 2$ to one-loop order in ChPT the distribution of $f$ is Gaussian

$$
\left\langle\delta\left(f-f^{\prime}\right)\right\rangle_{N_{f}}=\frac{1}{\sigma_{f} \sqrt{2 \pi}} e^{-\frac{\left(f-N_{f} E_{f} / 2\right)^{2}}{2 \sigma_{f}^{2}}} .
$$

where we have given the result for an arbitrary number of flavors $N_{f}$. Note that $E_{f}$ and $\sigma_{f}$ do not depend on $N_{f}$, see [2].

\section{QCD in one Euclidean dimension}

Consider now $1 \mathrm{dQCD}$ with a massive staggered fermion and gauge group $U\left(N_{f}\right)$

$$
Z_{N_{f}}\left(\mu_{c}, \mu\right)=\int_{U\left(N_{c}\right)} d U \operatorname{det} M
$$

where

$$
\operatorname{det} M=2^{-n N_{c}} \operatorname{det}\left[e^{n \mu_{c}}+e^{-n \mu_{c}}+e^{n \mu} U+e^{-n \mu} U^{\dagger}\right], \quad U \in U\left(N_{c}\right)
$$

and $d U$ is the Haar measure. The group integral can be solved analytically [14, 15], which shows explicitly that the partition function does not depend on $\mu$, as expected since there are no baryons 

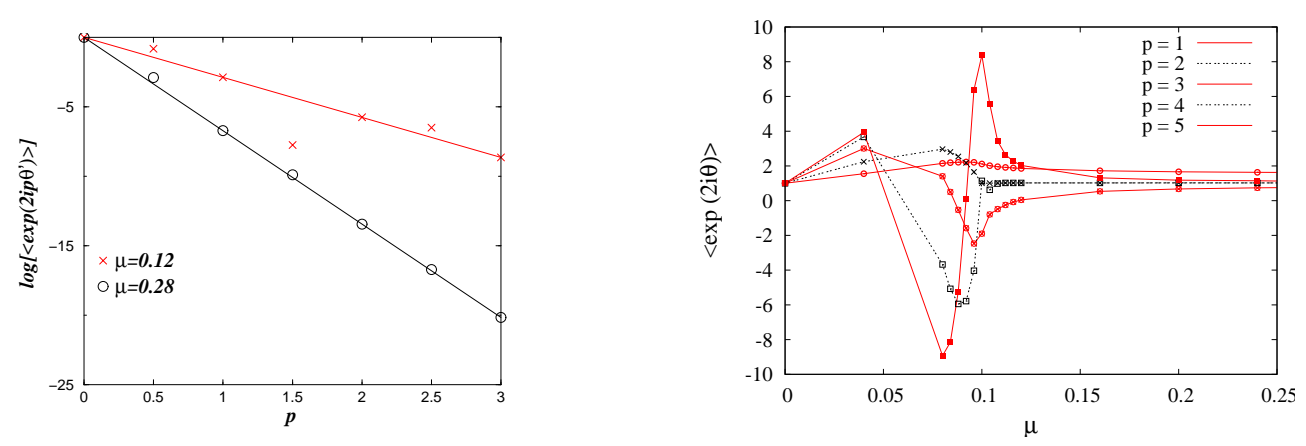

Figure 2: $U(3)$ lattice QCD in one dimension for $m_{q}=0.1$ i.e. $\mu_{c}=0.1$. Left we show the logarithm of the $p$-th moment as a function of $p$, for $\mu=(0.12,0.28)>\mu_{c}$, showing the expected linear behavior Eq. (3.4). The moments themselves as a function of $\mu$ for $p=1$ (the smoothest) to $p=5$ (right panel), show a sharp change at $\mu=\mu_{c}$ leading to the qualitative modifications of the $\theta$ distribution function described in the text. Note also the different behaviour of even (black symbols) and odd (red) moments, respectively (right).

in a $U\left(N_{c}\right)$ model. However, the quenched model has a transition to a pion condensed phase at $\mu=\mu_{c}$. So it resembles ordinary QCD with $\mu<\mu_{B} / 3$, in contrast to $S U\left(N_{c}\right)$ in one dimension where the transition of the quenched and the full model are very close to each other (which is of course the reason why we decided to work in $U(3)$ rather than in $S U(3)$ ).

Again the $\theta$ distribution follows form the moments

$$
\left\langle e^{2 i p \theta^{\prime}}\right\rangle=\int_{U\left(N_{c}\right)} d U \frac{\operatorname{det}^{p} M}{\operatorname{det}^{p} M^{\dagger}}
$$

For $\mu<m_{\pi} / 2$ we obtain

$$
\left\langle e^{2 i p \theta^{\prime}}\right\rangle=\left\langle e^{2 i \theta^{\prime}}\right\rangle^{p^{2}}=\left(1-\frac{\mu^{2}}{\mu_{c}^{2}}\right)^{p^{2}}
$$

With $\Omega \equiv-\log \left(1-\mu^{2} / \mu_{c}^{2}\right)$ this yields the quenched distribution

$$
\left\langle\delta\left(\theta-\theta^{\prime}\right)\right\rangle=\frac{1}{\sqrt{\pi \Omega}} e^{-\theta^{2} / \Omega} \quad \text { for } \quad \mu<\mu_{c}, N_{c} \rightarrow \infty,
$$

valid for $2 \theta \in[-\infty, \infty]$.

For $\mu>\mu_{c}$ the moments can be computed exactly for any $N_{c}$. They are

$$
\left\langle e^{2 i p \theta^{\prime}}\right\rangle=e^{-2 n|p| N_{c} \mu},
$$

and the quenched $\theta$ distribution again becomes a periodicised Lorentzian

$$
\left\langle\delta\left(2 \theta-2 \theta^{\prime}\right)\right\rangle=\frac{1}{\pi} \frac{\sinh \left(2 n N_{c} \mu\right)}{\cosh \left(2 n N_{c} \mu\right)-\cos (2 \theta)} \quad \text { for } \quad \mu>\mu_{c}, 2 \theta \in[-\pi, \pi] .
$$

The results have been verified numerically with a good accuracy, see Figure 2 . 


\section{Summary, and comments}

We have studied the $\theta$ distribution and related properties within chiral perturbation theory and one dimensional QCD. The qualitative features of the results are the same. In either case the baryons are omitted by fiat, and despite the absence of baryons there is a highly nontrivial $\mu$ dependence of these distributions in either cases.

Our main observation concerns the non trivial changes of the $\theta$ distribution when the chemical potential $\mu$ exceeds the quenched threshold for pion condensation $\mu_{c}=m_{\pi} / 2$, it can be summarised as follows:

- $\mu<m_{\pi} / 2$, i.e. quark mass outside the eigenvalues: Gaussian $\theta$ distribution.

$$
\left\langle\delta\left(\theta-\theta^{\prime}\right)\right\rangle=\frac{1}{\sqrt{\pi \Delta G_{0}}} \sum_{n=-\infty}^{\infty} e^{-(\theta+2 \pi n)^{2} / \Delta G_{0}}
$$

- $\mu>m_{\pi} / 2$, i.e. quark mass inside the eigenvalues: Lorentzian $\theta$ distribution.

$$
\left\langle\delta\left(2 \theta-2 \theta^{\prime}\right)\right\rangle=\frac{1}{\pi} \frac{\sinh \left(V L_{B}\right)}{\cosh \left(V L_{B}\right)-\cos (2 \theta)} .
$$

We reiterate that $\mu_{c}=m_{\pi} / 2$ is not a critical point of the full theory [16], and any sharp change at this unphysical threshold implies almost automatically specific difficulties in numerical approaches relying on extrapolation from low chemical potential values.

Further comments concern the validity of the central limit theorem: for $\mu<m_{\pi} / 2$ the distribution is Gaussian, thus fulfilling the conditions of the central limit theorem, and in agreement with the behaviour found in lattice simulations. However, the Lorentzian shape of the distribution of the phase valid for larger values of the chemical potential shows that one should not take for granted that the conditions for the central limit theorem are satisfied.

The analytic results show that exponentially large cancellations may take place when integrating over $\theta$, needed to measure correctly the baryon number. The extreme tail contributes significantly to the results. A small non Gaussian term in the tail of the $\theta$-distribution therefore could be the dominant term after integration over $\theta$. The precise form of this tail is of course difficult to access numerically.

In QCD in one Euclidean dimension the same behaviour has been observed by a direct evaluation of the involved partition functions, either analytically or numerically. We have studied in detail the behaviour of the moments of the distribution. The change of the distribution from Gaussian to Lorentzian is clearly seen in the sharp change of the momenta at $\mu_{c}$. In the same simple model, the distribution of the gluonic observables can be studied numerically. The results for the distributions of the plaquette - to be reported elsewhere [20] - can be used to demonstrate the effectiveness of the Fodor-Katz re-weighting[3]: we have explicitly shown that the usage of configurations generated at high temperatures maximises the overlap with a cold, dense target ensemble.

We close with a few general comments: the validity of the Gaussian assumption of the distributions are limited $\mu<m_{\pi} / 2$ : Hence, the point $\mu=m_{\pi} / 2$ looks more dangerous after this study: qualitative changes of the $\theta$ distribution, discontinuity of momenta. 
This rather pessimistic outcome might be partially mitigated by two observations: first, the $\theta$ distribution might be particularly difficult - experiments with plaquette or other observables in simpler models might be useful and give a different insight.

Secondly, and perhaps more significantly, the results of this study do not include baryons. The physical motivations for the success of any practical method for circumventing the sign problem relies on baryonic fluctuations $[5,6]$. One way to see this is to consider the average phase factor from Taylor expansion [4]:

$$
\left\langle e^{2 i \theta}\right\rangle_{1+1^{*}}=e^{L^{3} T\left(c_{2}-c_{2}^{I}\right) \mu^{2}}
$$

or, perhaps more simply, its log-derivative:

$$
\frac{\partial}{\partial \mu} \log \left\langle e^{2 i \theta}\right\rangle_{1+1^{*}}=\frac{\partial}{\partial \mu} \log Z_{1+1}-\frac{\partial}{\partial \mu} \log Z_{1+1^{*}} \propto\left(n_{B}(\mu)-n_{I}(\mu)\right)
$$

which equals the off diagonal susceptibility, and indicates an interplay between the sign problem, the temperature, and the baryonic contributions [17], [18]. Current and future work will address this issue $[19,20]$.

\section{References}

[1] Ph. de Forcrand, plenary talk at Lattice09, this Volume; M. P. Lombardo, J. Phys. G 35 (2008) 104019

[2] M. P. Lombardo, K. Splittorff and J. J. M. Verbaarschot, Phys. Rev. D 80, 054509 (2009)

[3] Z. Fodor and S. D. Katz, JHEP 0203 (2002) 014

[4] C. R. Allton et al. , Phys. Rev. D 71, 054508 (2005)

[5] M. Alford, A. Kapustin, and F. Wilczek, Phys. Rev. D 59 (1999) 054502.

[6] M. P. Lombardo, Nucl. Phys. Proc. Suppl. 83 (2000) 375

[7] P. de Forcrand and O. Philipsen, Nucl. Phys. B 642 (2002) 290

[8] M. D'Elia and M. P. Lombardo, Phys. Rev. D 67 (2003) 014505

[9] J. Ambjorn, K. N. Anagnostopoulos, J. Nishimura and J. J. M. Verbaarschot, JHEP 0210, 062 (2002)

[10] Z. Fodor, S. D. Katz and C. Schmidt, JHEP 0703, 121 (2007)

[11] K. Splittorff and J. J. M. Verbaarschot, Phys. Rev. D 77, 01451

[12] S. Ejiri, Phys. Rev. D 77, 014508 (2008)

[13] S. Ejiri et al. [WHOT-QCD Collaboration], arXiv:0909.2121 [hep-lat]

[14] N. Bilic and K. Demeterfi, Phys. Lett. B, 212 (1988) 83

[15] L. Ravagli and J. J. M. Verbaarschot, Phys. Rev. D 76 (2007) 054506

[16] K. Splittorff, PoS LAT2006, 023 (2006)

[17] R. V. Gavai and S. Gupta, Phys. Rev. D 68, 034506 (2003)

[18] M. D’Elia and F. Sanfilippo, Phys. Rev. D 80 (2009) 014502

[19] M. P. Lombardo, K. Splittorff and J. J. M. Verbaarschot, arXiv:0910.5482 [hep-lat].

[20] M. P. Lombardo, K. Splittorff and J. J. M. Verbaarschot, In preparation. 